\title{
Hybrid Electrochemical/Chemical Synthesis of Quantum Dots
}

\author{
REGINALD M. PENNER* \\ Institute for Surface and Interface Science, \\ Department of Chemistry, University of California, \\ Irvine, California 92679-2025 \\ Received August 25, 1999
}

\section{ABSTRACT}

The "electrochemical/chemical method" (or "E/C method") is a new wet chemical method for synthesizing semiconductor quantum dots on graphite surfaces. The $\mathrm{E} / \mathrm{C}$ synthesis of quantum dots composed of the generic semiconducting salt, $M X$, typically involves three steps: (1) electrochemical deposition of nanoparticles of the metal, $\mathrm{M}^{\circ}$, from a solution of metal ions, $\mathrm{M}^{\mathrm{n}+} ;(2)$ electrochemical oxidation of these metal particles to $\mathrm{MO}_{\mathrm{n} / 2}$, and; (3) displacement of the oxygen from $\mathrm{MO}_{\mathrm{n} / 2}$ using $\mathrm{HX}$ (for example) to yield nanoparticles of MX. This conversion from metal to metal oxide to metal salt occurs on a particle-by-particle basis; that is, each metal nanoparticle is converted into a semiconductor nanoparticle. E/C-synthesized $\beta$-Cul and CdS quantum dots possess many of the attributes of quantum dots synthesized using molecular beam epitaxy, including epitaxial orientation on the graphite surface, a narrow size dispersion, and strong, particle size-tunable photoluminescence. However, the E/C method is faster, cheaper, and applicable to a greater number of materials.

\section{Introduction}

The remarkable size-dependent optical properties of semiconductor nanocrystals or quantum dots were discovered in the early 1980s by the research groups of Brus, $^{1,2}$ Henglein, ${ }^{3,4}$ and Grätzel. ${ }^{5}$ In this pioneering work, liquid-phase methods for synthesizing suspensions of nanocrystals in solvents such as water and acetonitrile were developed (Scheme 1a).

In the ensuing 15 years, synthetic methods for quantum dots have been refined, and it is now possible to obtain nanocrystals of I-VII materials (e.g., $\mathrm{AgBr}^{6,7}$ ), II-VI materials (e.g., $\mathrm{CdSe}^{8}$ ), III-V materials (e.g., $\mathrm{GaAs}^{9,10}$ ), and Group IV materials (e.g., $\mathrm{Si}^{11}$ ) that are narrowly dispersed in diameter. Suspensions of these nanocrystals are stable, and these suspensions have proven to be ideal for investigating the photophysics of quantum dots.

The discovery in 1990 of the growth by molecular beam epitaxy (or MBE) of germanium islands on silicon, ${ }^{12,13}$ and indium gallium arsenide (i.e., $\operatorname{In}_{x} \mathrm{Ga}_{1-x} \mathrm{As}$ ) islands on gallium arsenide, ${ }^{14}$ constituted a breakthrough with re-

Reg Penner obtained B.A. degrees in chemistry and biology from Gustavus Adolphus College in St. Peter, M N, in 1983. He studied electrochemistry with Chuck Martin at Texas A\&M and graduated with a Ph.D. in 1987. For the next two years, he was a postdoctoral researcher with Nate Lew is first at Stanford University and later at Caltech. During this period, he carried out investigations focusing on ultramicroelectrodes, cold fusion, and scanning probe microscopy. In 1990, he joined the faculty at UCI where he is currently a professor of chemistry. His research group develops new methods for synthesizing and characterizing nanomaterials (including metals, polymers, and semiconductors) on electrode surfaces. He is a NSF Young Investigator, an ONR Young Investigator, and a Beckman Foundation Young Investigator as well as an A.P. Sloan Fellow and a Camille Dreyfus Teacher-Scholar.

78 ACCOUNTS OF CHEM ICAL RESEARCH / VOL. 33, NO. 2, 2000
Scheme 1. Three Methods for Synthesizing Semiconductor Quantum Dots

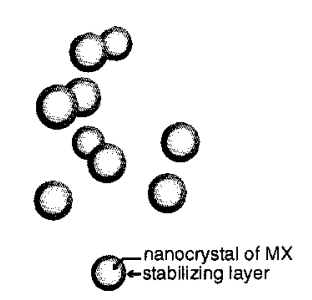

a. Solution phase synthesized MX nanocrystals

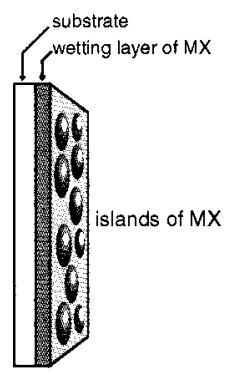

b. Stranski-Krastanov Growth of MX Islands by MBE

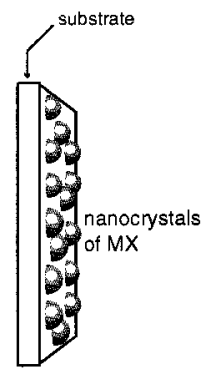

c. E/C synthesized $\mathrm{MX}$ nanocrystals spect to the assimilation of quantum dots into technology (Scheme 1b). When quantum dots are "synthesized" using molecular beam epitaxy, the crystalline lattice of each quantum dot is assembled in an atomic layer-by-layer fashion. Quantum dots prepared by molecular beam epitaxy possess two important properties: the crystalline lattices of each island are identically oriented with respect to the sample surface (i.e., the growth of these islands is "epitaxial"), ${ }^{15}$ and an intimate electrical contact exists between the island and the conductive sample surface. Recently, these two attributes have facilitated the incorporation of quantum dots into laser diodes (LDs). ${ }^{16}$ Unfortunately, molecular beam epitaxy is limited in terms of the materials from which quantum dots can be obtained, and in terms of the accessible island diameter, island thickness, and the number density of islands on the surface.

We have sought to develop a solution-phase method for synthesizing supported quantum dots (Scheme 1c). Ideally, these quantum dots would possesses all of the attributes of those prepared using liquid-phase synthetic methods (adjustibility of the nanocrystal identity and diameter; excellent size monodispersity) and the technological utility of quantum dots prepared by molecular beam epitaxy (specifically, the deposition of nanocrystals with a defined orientation and an electrical contact to the outside world). For two systems ( $\beta$-Cul, and CdS), we have demonstrated that a radically new method can produce quantum dots possessing all of these attributes. We call this new method the electrochemical/chemical (or E/C) method.

The E/C synthesis of nanocrystals of a metal salt, MX, involves three steps, as shown in Scheme 2: first, metal nanocrystals (e.g., cadmium) that are similar in size are electrochemically deposited on a graphite surface from an aqueous solution containing the metal ion (e.g., $\mathrm{Cd}^{2+}$ ); second, these particles are oxidized either chemically or electrochemically (this transforms the $\mathrm{Cd}$ particles into $\mathrm{Cd}(\mathrm{OH})_{2}$ particles, for example); and finally, the hydroxyl $\left(\mathrm{OH}^{-}\right)$is displaced with another anion (e.g., sulfide; $\mathrm{S}^{2-}$ ) to yield nanoparticles of a semiconducting salt (e.g.,

\footnotetext{
*E-mail: rmpenner@uci.edu.
} Published on Web 01/19/2000 
Scheme 2. E/C Synthesis Schemes for Three Semiconducting Materials
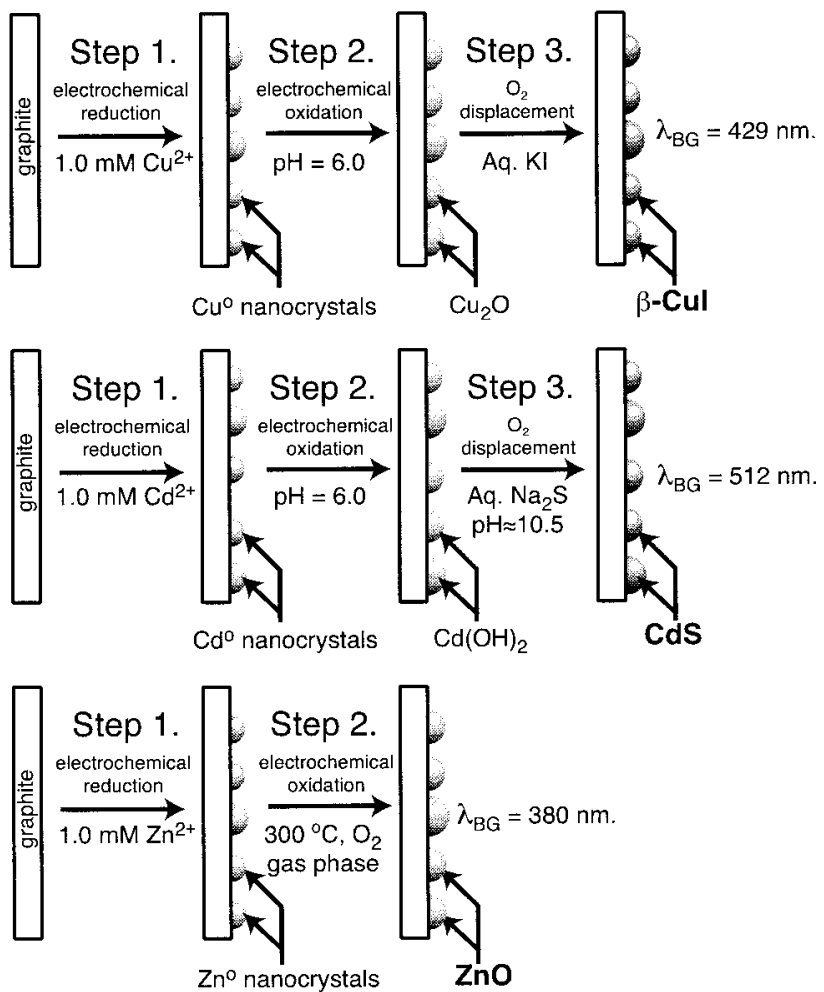

cadmium sulfide, CdS). In fact, billions of CdS quantum dots are produced on the graphite surface in a single E/C synthesis operation-one CdS particle for every cadmium metal nanoparticle that was formed in step 1. Typical atomic force microscope images of CdS quantum dots are shown in Figure 1.

In this Account, the $\mathrm{E} / \mathrm{C}$ synthesis of $\mathrm{ZnO}, \mathrm{Cul}$, and $\mathrm{CdS}$ is reviewed, the photoluminescence properties of E/Csynthesized quantum dots are summarized, a method for improving particle size monodispersity is presented, and the construction of a photodetector based on E/Csynthesized nanocrystals is briefly described.

\section{Electrodeposition of Size-Monodisperse Metal Nanoparticles}

As shown in Scheme 2 , the $\mathrm{E} / \mathrm{C}$ syntheses of $\mathrm{ZnO}, \beta$-Cul, and $\mathrm{CdS}$ begins with the electrodeposition of zinc, copper, and cadmium nanoparticles, respectively. The reason this first step is especially important is implicit in Schemes 1 and 2: the chemical transformations from metal to metal oxide to metal salt take place on a particle-by-particle basis. In the E/C synthesis of CdS nanocrystals, for example, each cadmium metal nanoparticle deposited in step 1 of the synthesis is oxidized in step 2 to yield a nanoparticle of $\mathrm{Cd}(\mathrm{OH})_{2}$, and each $\mathrm{Cd}(\mathrm{OH})_{2}$ nanoparticle reacts in step 3 to yield a nanocrystal of CdS. This CdS nanocrystal contains the same number of cadmium atoms as were present in the "parent" cadmium metal nanoparticle deposited in step 1 of the synthesis; however, its diameter will be larger as a consequence of the larger

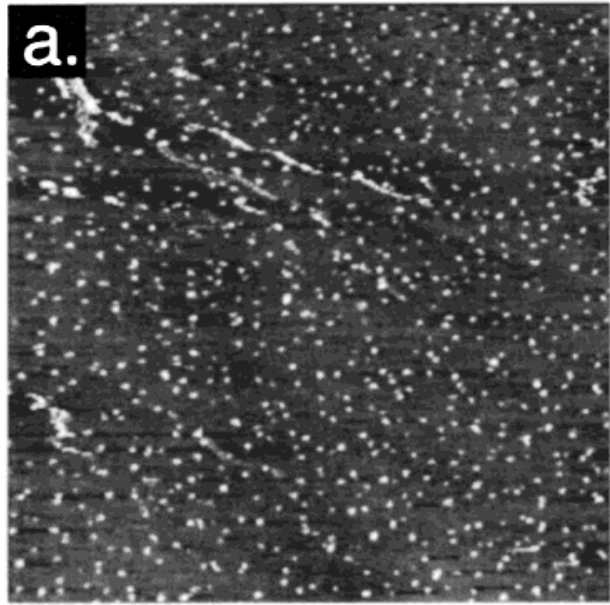

b.

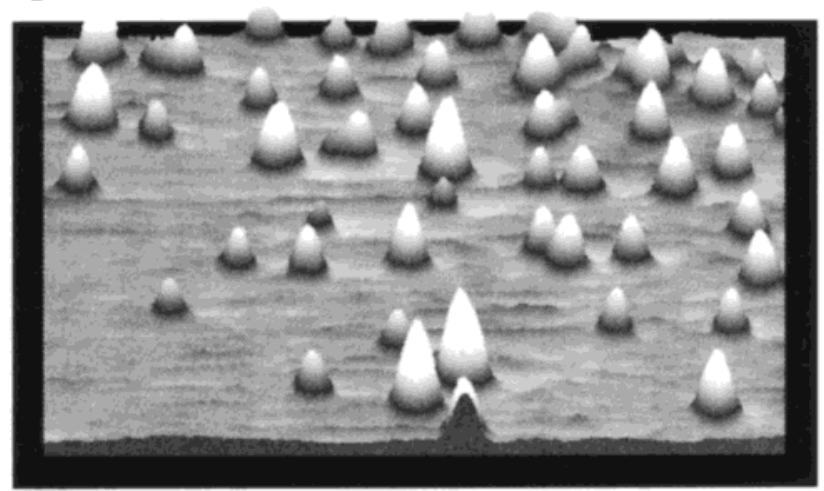

FIGURE 1. Noncontact atomic force microscope (nanocrystal-AFM) images of graphite surfaces following the electrochemical/chemical synthesis of sulfur-capped CdS nanocrystals. The size dispersion evident-particularly in (b)-derives partially from the nonuniformity of the thickness of the sulfur capping layer. (a) A 3- $\times 3-\mu \mathrm{m}$ image of CdS nanocrystals having a mean core diameter of $60 \AA$. (b) Perspective view of $C d S$ nanocrystals having a mean core diameter of $75 \AA$.

molar volume of CdS $\left(29.98 \mathrm{~cm}^{3} \mathrm{~mol}^{-1}\right.$ for CdS vs 13.01 $\mathrm{cm}^{3} \mathrm{~mol}^{-1}$ for $\mathrm{Cd}^{\circ}$ ). The key point is that in an $\mathrm{E} / \mathrm{C}$ synthesis, the size and size dispersion of product nanoparticles are decided by the corresponding properties of the metal nanoparticles deposited in step 1 , and this step therefore assumes special importance.

An electrochemical method for preparing metal nanoparticles on graphite surfaces was discovered in 1996 by Jim Zoval. ${ }^{17}$ Zoval found that the electrodeposition of silver onto a graphite electrode surface yielded silver nanoparticles, even if minute quantities of silver of less than 1 equivalent atomic layer were deposited. Surface scientists refer to this prompt three-dimensional growth mode as Volmer-Weber deposition. ${ }^{18}$ Since 1996, we have found that other metals, including platinum, ${ }^{19}$ copper, ${ }^{20}$ nickel, ${ }^{21}$ cadmium, ${ }^{22,23}$ and zinc, ${ }^{24}$ al so deposit by this same VolmerWeber mechanism on graphite. Noncontact atomic force microscope (NC-AFM) images of a graphite surface following the deposition of 0.039 monolayer of platinum, for example, are shown in Figure 2a. ${ }^{19}$ Platinum nanoparticles 

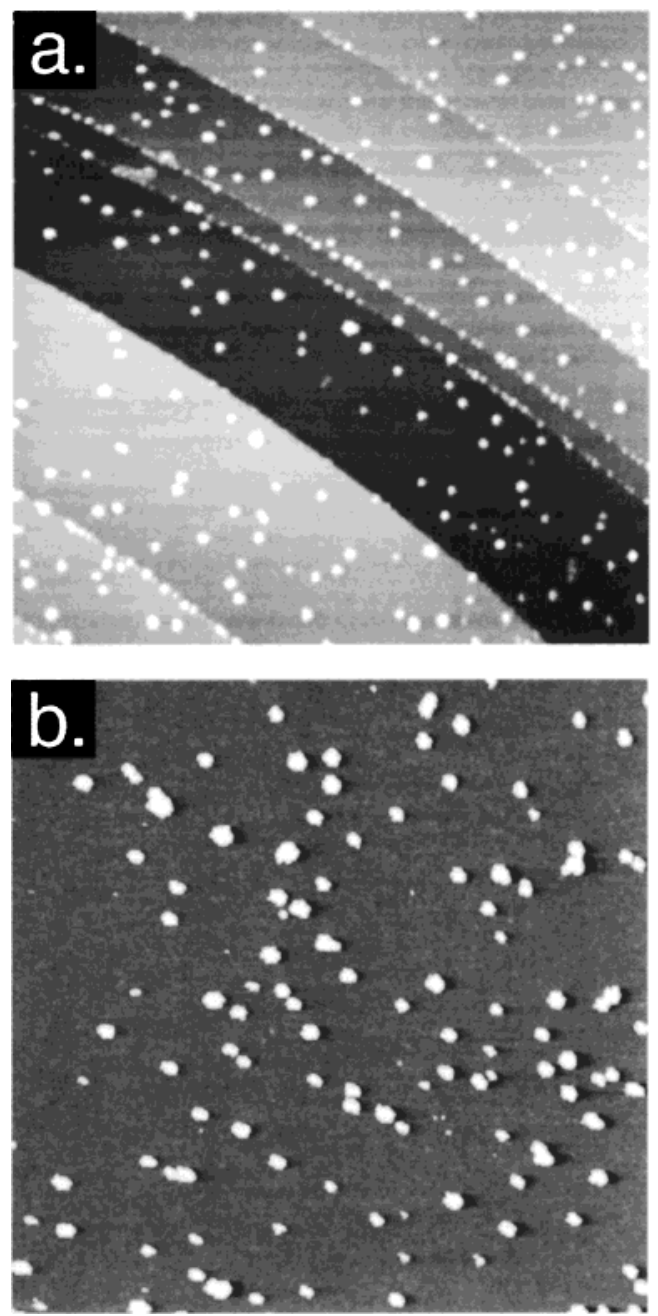

FIGURE 2. Noncontact atomic force microscope (NC-AFM) images of graphite and silicon surfaces following the electrodeposition of metal nanoparticles. (a) A 3- $\times 3-\mu \mathrm{m}$ image of platinum nanocrystals having a mean diameter of $52 \AA$ on graphite. (b) A 3- $\times 3-\mu$ m image of silver nanocrystals having a mean diameter of $75 \AA$ on Si(100).

having a mean diameter of $52 \AA$ are seen on a graphite surface in this image. The image of Figure $2 a$ also shows that platinum nanoparticles have nucleated both at defect sites such as step edges, and on atomically smooth terraces. Volmer-Weber growth is favored at coordinatively saturated surfaces, like graphite, that are character-

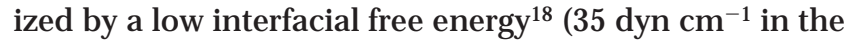
case of the graphite basal plane ${ }^{25}$ ). Becca Stiger has demonstrated ${ }^{26}$ that the Volmer-Weber deposition mechanism also operates at another low-energy surface: that of hydrogen-terminated $\mathrm{Si}(100)$. The NC-AFM image of Figure 2c, for example, shows a H-terminated $\mathrm{Si}(100)$ surface following the electrodeposition of 0.037 equivalent atomic layers of silver from an acetonitrile-based plating solution. ${ }^{26}$

The mean diameter of electrodeposited metal nanoparticles on graphite or silicon increases as a function of the deposition time. This provides a means by which quantum dots of any desired mean diameter may be prepared.

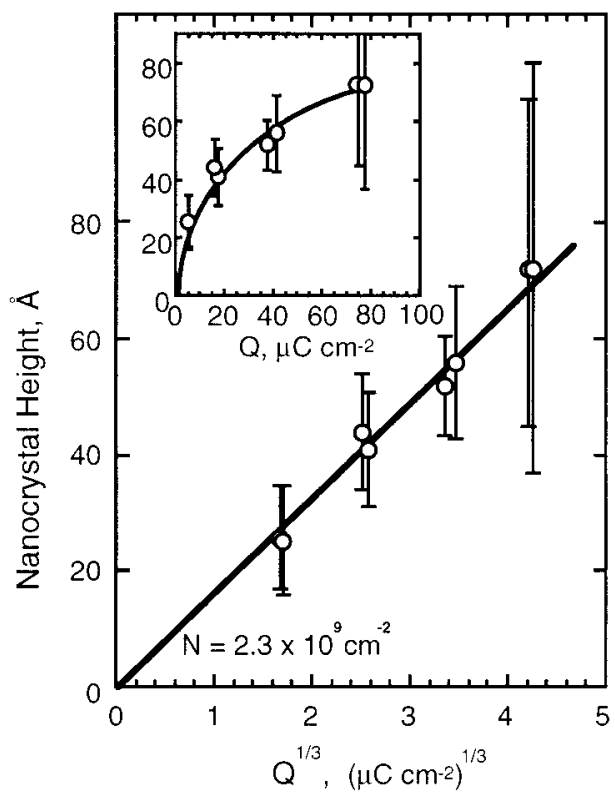

FIGURE 3. Plots of the mean particle height versus charge (inset), and the mean particle height versus the inverse cube root of the deposition charge density, $Q^{1 / 3}$ (in units of $\mu$ Coulomb/ $\left.\mathrm{cm}^{2}\right)^{1 / 3}$, for the electrodeposition of platinum nanocrystals on graphite. The straight line shown here is that expected for a particle density of $2.3 \times 10^{9}$ $\mathrm{cm}^{-2}$. The potentiostatic deposition of platinum in these experiments was carried out using an aqueous plating solution containing 1.0 $\mathrm{mM} \mathrm{PtCl}{ }^{2-}$ and $0.10 \mathrm{M} \mathrm{HCl}$. The deposition potential was $-600 \mathrm{mV}$ vs a saturated mercurous sulfate reference electrode. (From ref 19.)

In the case of platinum, for example, Figure 3 shows that the mean particle diameter increases in proportion to the cube root of the quantity of platinum. ${ }^{19}$

\section{Conversion of Metal Nanoparticles to Semiconductor Nanoparticles}

In 1997, a graduate student in our group, Gregor Hsiao, discovered that nanocrystals of copper(I) iodide were produced on graphite surfaces following the electrodeposition of copper and the exposure of these surfaces to aqueous iodide-containing solutions. His E/C synthesis of Cul sparked our interest in this unique synthetic method. The initial assignment of Cul to the product of his synthesis was based on the observation of a bright violet photoluminescence $(\mathrm{PL})$ from these surfaces at room temperature. It was assumed that the violet PL was caused by band-edge emission from $\gamma$-Cul having a direct band gap at $2.92 \mathrm{eV}$ (corresponding to violet emission at $429 \mathrm{~nm}$ ). How was the crystal structure of these nanocrystals measured, and how was the existence of a cuprous oxide intermediate deduced?

In 1997, these questions were difficult ones for us to answer. In the specific case of Cul, for example, we had no direct structural evidence for Cul on these surfaces, and no reason to suspect that $\mathrm{Cu}_{2} \mathrm{O}$ might be an intermediate in the synthesis of Cul, as shown in Scheme 2. The characterization of these nanocrystals using a diffraction-based method was required in order to learn their structure, but the submonolayer coverage of Cul nanoparticles on these surfaces was insufficient to permit 


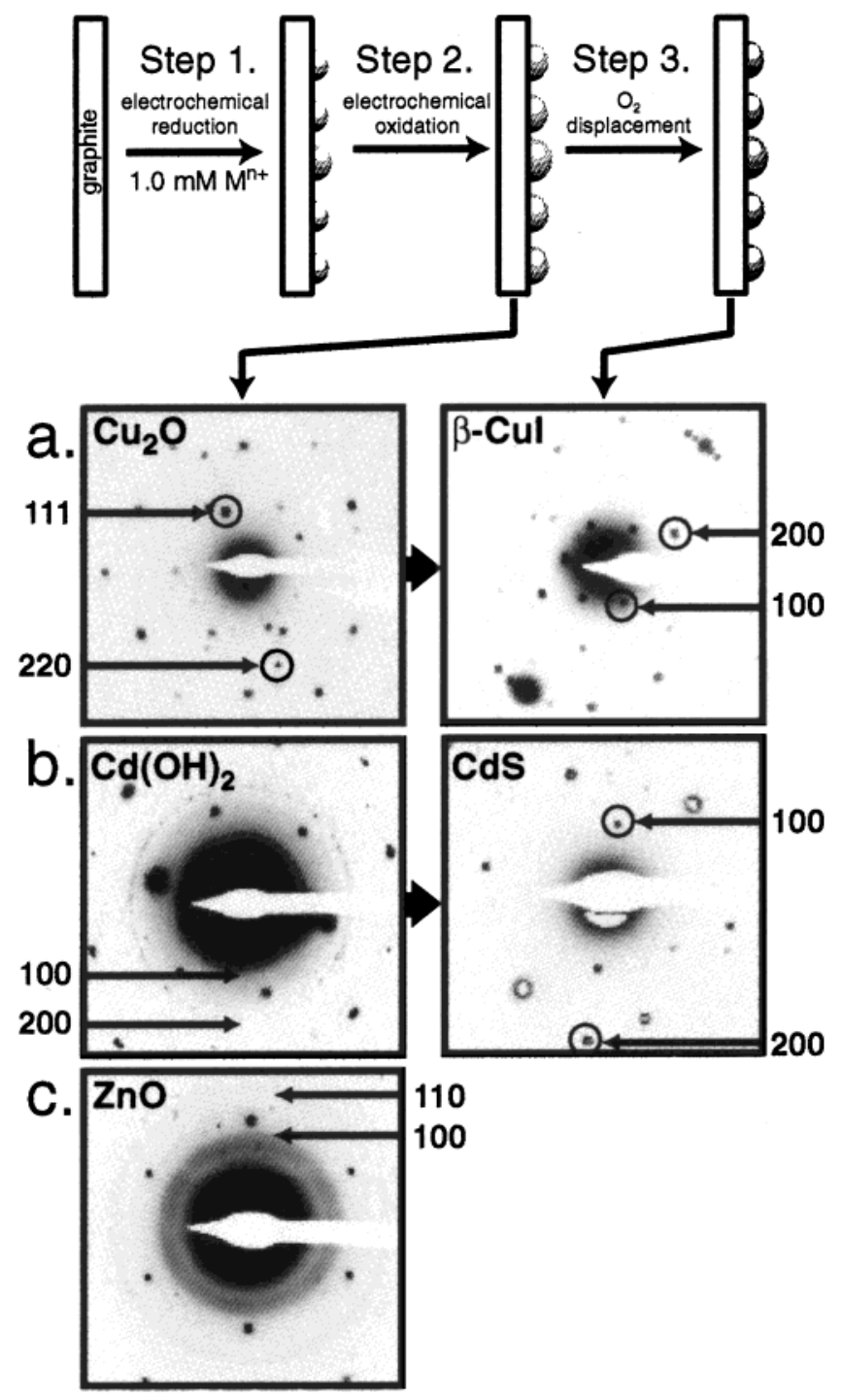

FIGURE 4. Selected area electron diffraction patterns (SAED) acquired for the $E / C$ synthesis of three materials: (a) $\beta$-cuprous iodide, (b) cadmium sulfide, and (c) zinc oxide. In each case, a 10$\mu \mathrm{m}$-diameter region of the surface was sampled. Assignments of one or two diffraction spots associated with each of these products are indicated. In addition, a hexagonal array of diffraction spots assignable to graphite is visible in each pattern.

powder X-ray diffraction to be used for this purpose. Fortunately, a postdoctoral member of our group, Sasha Gorer, discovered that the E/C synthesis of Cul nanocrystals could be carried out on very thin ( $\sim 300 \AA$ thick) graphite flakes, and that selected area electron diffraction (or SAED) could be employed to analyze the composition and orientation of these nanocrystals. His SAED analysis, summarized in Figure $4 \mathrm{a}$, showed that nanoparticles produced by copper electrodeposition in an aqueous electrolyte were composed of cubic $\mathrm{Cu}_{2} \mathrm{O}$, not metallic copper. ${ }^{20}$ Thus, the E/C synthesis of Cul proceeded via an intermediate, as shown in Scheme 2. Gorer's SAED analysis yielded three other surprises: First, "Cul" was, in fact, $\beta$-Cul-a wurzite phase allotrope of this compound that is thermodynamically stable only at temperatures greater than $300^{\circ} \mathrm{C}-$ not $\gamma$-Cul which is the allotrope that is stable at room temperature. $\mathrm{Second}, \mathrm{Cu}_{2} \mathrm{O}$ nanoparticles did not move laterally on the surface during conversion to Cul. When coupled with the diffraction data, this observation justified the conclusion that the conversion to $\mathrm{Cul}$ from $\mathrm{Cu}_{2} \mathrm{O}$ was occurring on a "particle-by-particle" basis (the alternative, involving the appearance of "new" particles of $\mathrm{Cul}$ and the disappearance of "old" $\mathrm{Cu}_{2} \mathrm{O}$ particles, was clearly not occurring). Finally, as shown in Figure $4 a$, SAED patterns for thousands of $\beta$-Cul nanocrystals consisted of spots, not rings.

Because these SAED patterns were derived from diffraction from a discontinuous layer of nanocrystals on the graphite surface, this result implied that most Cul nanocrystals on these surfaces adopted a preferred, and crystallographically identical, orientation with respect to the hexagonal graphite lattice. In other words, the Cul nanocrystals were epitaxially deposited. A year later, Matt Anderson showed that nanocrystals of CdS (a II-VI material) could be synthesized using an exactly analogous procedure (Scheme 2 ) involving the electrodeposition of $\mathrm{Cd}^{\circ}$, oxidation to $\mathrm{Cd}(\mathrm{OH})_{2}$, and exposure of oxide nanoparticles to basic, aqueous $\mathrm{S}^{2-} .22$ Nanocrystals of wurtzite $\mathrm{CdS}$ also deposited in an epitaxial fashion on the graphite surface $^{22}$ (see Figure 4b).

The preference of Cul and CdS nanocrystals for a particular orientation on graphite is explained by the plots shown in Figure 5. In this figure,the energy of islands having a particular (as indicated) and azimuthal orientation on a graphite surface has been calculated. All three of these materials possess the wurtzite crystal structure, and we have assumed in our calculation that the (0001) plane of the crystal contacts the hexagonal graphite surface. Data for platinum, Cul, CdS, and ZnO are shown, and it is apparent that the plots for Cul and CdS, but not $\mathrm{ZnO}$, exhibit prominent minima in the energy versus angle calculation. These energetic wells correspond to coincidences between the lattice of the nanocrystal and that of the graphite surface, as shown schematically for CdS at the bottom of Figure $5.23 \mathrm{In}$ contrast, no significant energetic minima are seen for the (0001) surface of wurtzite-phase $\mathrm{ZnO}$, indicating that this interface is badly mismatched at all angles. ${ }^{24}$ Thus, no energetic motivation exists for ZnO nanocrystals to adopt a preferential orientation on the graphite surface, and a SAED powder pattern like that seen in the experimental data of Figure $4 \mathrm{c}$ is expected. Data for the three low-index faces of platinum are also shown in Figure 4 for purposes of comparison. Especially for [100] and [111] (the likely termination faces for a platinum nanocrystal), pronounced energy minima are absent. Experimentally, we have found that platinum nanocrystals, like nanocrystals of $\mathrm{ZnO}$, have no orientational preference (data not shown). ${ }^{19}$ At least for these four materials, the relatively simple calculation used to generate the data of Figure 5 correctly predicts the epitaxial nature of the $\mathrm{E} / \mathrm{C}$ deposition for both $\beta$-Cul and CdS, and it predicts the absence of epitaxy for nanocrystals of $\mathrm{ZnO}$ and platinum on graphite.

Although both Cul and CdS possess an excellent lattice match to graphite, it is nevertheless unclear how nanocrystals of these materials locate the minimum energy 


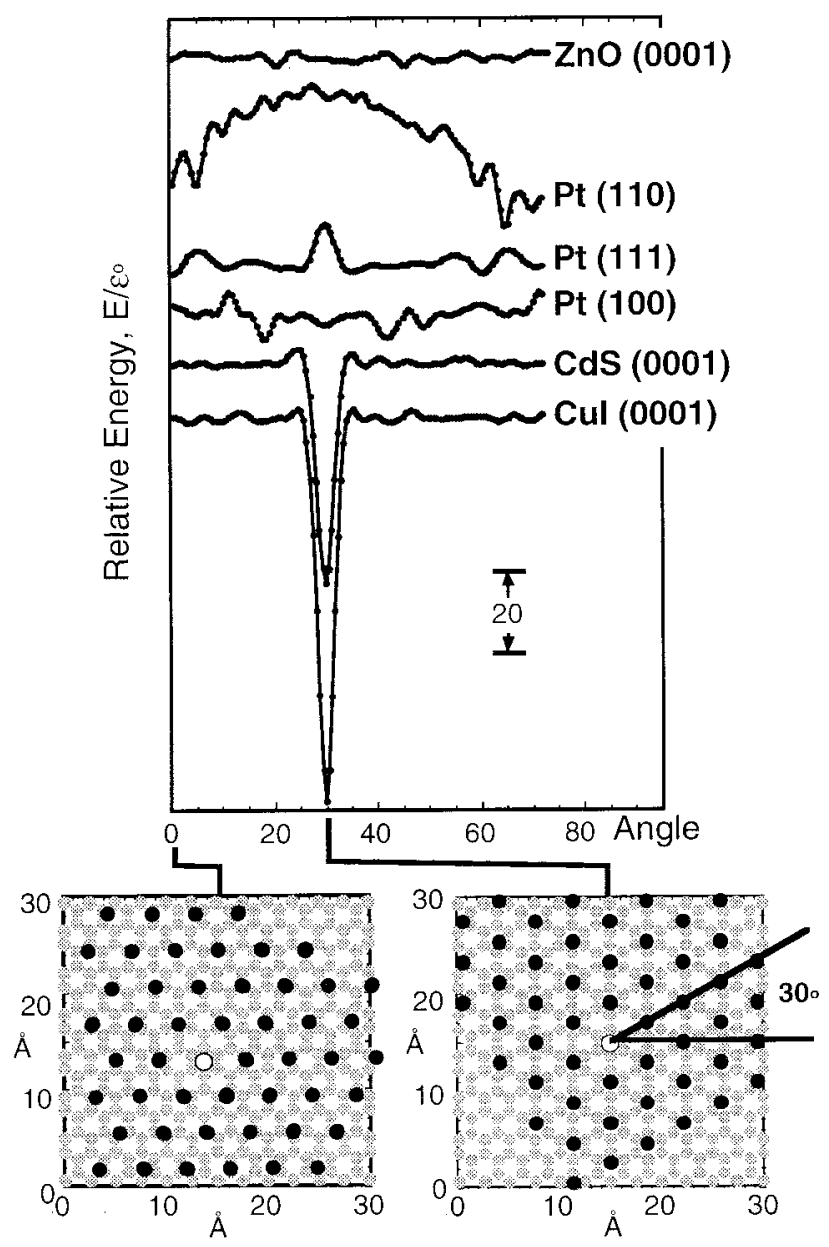

FIGURE 5. Plot of the calculated interfacial energy, $E / \epsilon_{0}$, versus angle for the interface of six different interfaces involving the graphite(0001) surface. In each of these calculations, the absolute interfacial energy, $E$, has been normalized by the well depth of the Lennard-J ones pairwise potential, $\epsilon_{0}$. Each island was approximately $25 \AA$ in diameter and consisted of 126 atoms partitioned into two layers of equal size. The graphite surface consisted of 1882 atoms also partitioned into two layers. At bottom left is shown the configuration for the $\mathrm{CdS}$-graphite calculation corresponding to $0^{\circ}$, and at bottom right is shown the configuration for this system corresponding to the energy minimum at $30^{\circ}$.

configurations shown in Figure 5 when no lateral motion of these particles is apparent in TEM images of these surfaces obtained before and following the third step of the synthesi $s^{20,23}$ Resolution of this mystery must await a full atomic level description of the displacement reaction which we hope to formulate in the future. Collectively, however, SAED data coupled with TEM images of $\beta$-Cul and CdS nanocrystals convincingly demonstrate that metal oxide nanoparticles are converted on a particle-byparticle basis into semiconducting metal salt particles, thereby supporting the "E/C paradigm" set forth in the schematic diagrams of Scheme 2.

\section{Photoluminescence Spectroscopy of E/C-Synthesized Materials}

Usually, the photoluminescence (or PL) of molecules located within a few angstroms of a conductive surface is
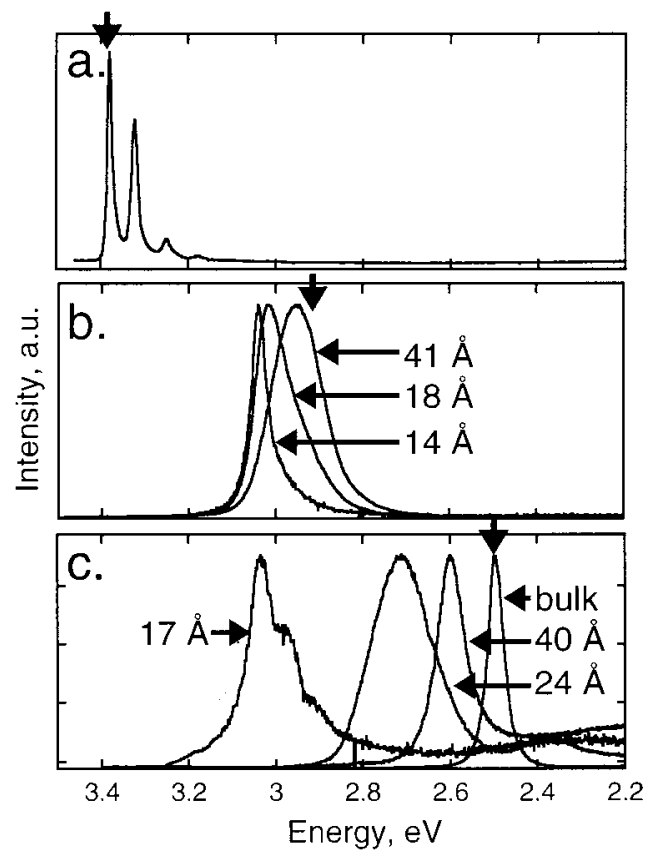

FIGURE 6. Photoluminescence (PL) emission spectra for E/Csynthesized materials. In all three cases, PL was excited using the 351-nm line of an $\mathrm{Ar}^{+}$laser. (a) ZnO film approximately $100 \AA$ in thickness; $T=20 \mathrm{~K}$. (b) $\beta$-Cul nanocrystals of three radii as labeled; $T=295 \mathrm{~K}$. (c) Sulfur-passivated CdS nanocrystals of three radii as labeled; $T=20 \mathrm{~K}$.

efficiently quenched. ${ }^{27}$ Remarkably, this is not the case for the PL of E/C-synthesized nanocrystals on graphite surfaces. ${ }^{28}$ Typical PL spectra for $\mathrm{ZnO},{ }^{24} \beta$-Cul, ${ }^{20}$ and $\mathrm{CdS},{ }^{23}$ for example, are shown in Figure 6.

Ralph Nyffenegger together with undergraduates Mo Shabaan and Ben Craft developed the abbreviated E/C synthesis for $\mathrm{ZnO}$ films and nanoparticles indicated in Scheme $2 .{ }^{24} \mathrm{~A} \mathrm{PL}$ spectrum for one of their $\mathrm{ZnO}$ films is shown in Figure $6 \mathrm{a}^{29}$ This spectrum is notable for two reasons. ${ }^{24}$ First, a progression of emission lines, instead of a single PL line, is observed in this spectrum. These additional lines derive from phonon loss transitions in which an integer number of phonons have been excited in the course of emission. The energy separating these emission lines ( $\sim 76 \mathrm{meV}$ ) corresponds to the energy of a single LO phonon in this material, and the presence of six phonon loss lines in this spectrum is consistent with an extremely low defect density for this material. Second, no emission is seen in the low-energy region of the PL spectrum in Figure 6a from 3.2 (UV) to $2.2 \mathrm{eV}$ (green). Emission from midgap states derived from impurities and defects has been a ubiquitous feature of the PL spectrum for $\mathrm{ZnO}$ synthesized using other methods. ${ }^{24}$ The absence of this emission in the spectrum of Figure 6a suggests that E/C-synthesized ZnO films are free of impurities (i.e., "intrinsic") and defects. ${ }^{30}$ Thus far, we have not investigated the optical properties of $\mathrm{ZnO}$ quantum dots because until recently, the means for photoexciting these particles at energies greater than $3.5 \mathrm{eV}$ did not exist in our laboratory.

PL spectra for $\beta$-Cul and CdS quantum dots are shown in Figure $6 b$ and $c$, respectively. Emission from E/C- 


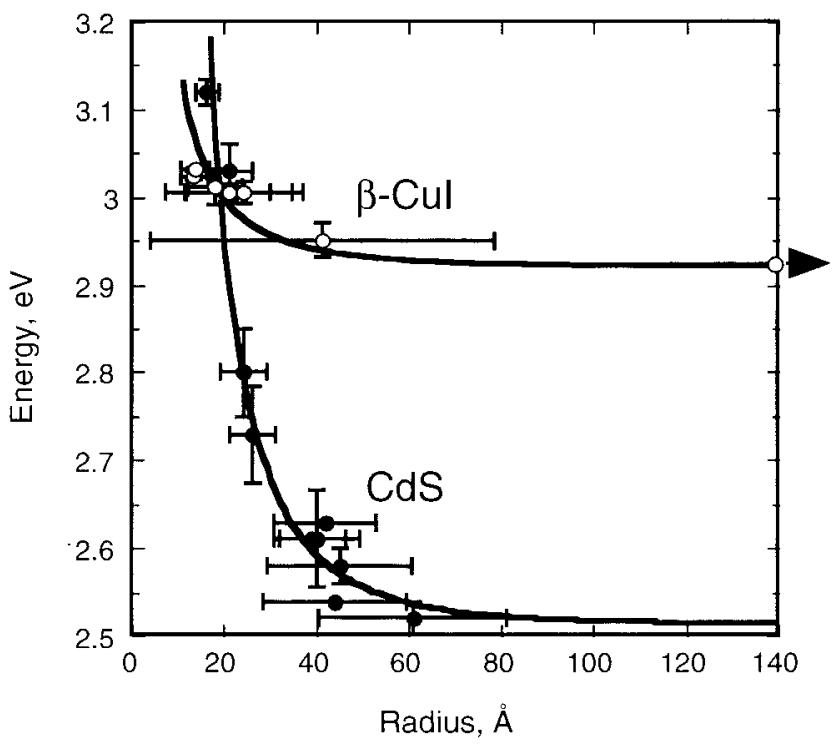

FIGURE 7. Plots of the energy of maximum emission versus the radius for nanocrystals of $\beta$-Cul (O) and $\mathrm{CdS}(\mathbf{O})$. Error bars represent the dispersion (i.e., $\pm 1 \sigma$ ) in the energy and the nanoparticle radius for a series of measurements at different locations on the graphite surface. Solid lines represent the calculated band gap energy as a function of radius for nanoparticles of these two materials.

synthesized nanocrystals of $\beta$-Cul and CdS are blue shifted from the bulk band gaps of these materials (indicated by arrows at the top of each spectrum), and the magnitude of this shift is inversely related to the nanoparticle radius. These spectral shifts are a manifestation of the confinement of photogenerated electrons and holes in these diminutive particles. ${ }^{31-33}$ The magnitudes of the spectral shifts seen for small CdS and $\beta$-Cul nanoparticles are disparate. For example, CdS nanocrystals having a mean radius of $17 \AA$ are blue-shifted by $\sim 500$ meV, whereas nanocrystals of $\beta$-Cul nanocrystals with radii of $13.6 \AA$ are blue-shifted by just $\sim 120 \mathrm{meV}$. The greater "sensitivity" of CdS to the nanocrystal radius derives from the smaller hole effective mass in this material $\left(0.8 m_{0}\right.$ vs $1.4 m_{0}$ for Cul $)$. Because the dielectric constants of $\beta$-Cul and CdS are similar (4.58 vs 5.4 ), the lighter holes of CdS possess larger wave functions than their counterparts in Cul, and the Bohr radius for an exciton in CdS is therefore proportionately larger.

Plots of PL energy versus radius for $\beta$-Cul and CdS are shown in Figure 7 (data points). Also plotted in Figure 7 are calculations of the band gap energy as a function of the nanocrystal radius for these two materials (solid lines). The agreement between the calculated band gap and the experimentally observed PL indicates that photoexcited electrons at the conduction band edge recombine directly with holes at the valence band edge. This process results in the emission of a PL photon having an energy equal to that of the band gap. This radiative recombination pathway competes with a second process involving the thermalization of electrons and holes into trap states existing within the band gap. These traps are the inevitable consequence of surface defects such as dangling bonds, and impurities. If the recombination of trapped electrons and holes produces photons, this process manifests itself as PL emission that is red-shifted from the band gap. Little evidence for this undesirable "trap state" emission exists in the spectra shown in Figure 6.

Collectively, the data shown in Figures 6 and 7 permit the following conclusions: (1) E/C-synthesized nanocrystals of $\beta$-Cul and CdS on graphite surfaces are luminescent. (2) Luminescence from these nanocrystals derives from the band-to-band recombination of photogenerated electron and holes; this transition does not involve "deep" trap states. This fact suggests that the crystal quality and purity of these materials are excellent. (3) The PL emission energy can be smoothly tuned by adjusting the nanocrystal radius using the $\mathrm{E} / \mathrm{C}$ synthesis conditions (i.e., the duration of metal plating in step 1).

\section{V. "Decoupling" for Improved Particle Size Monodispersity}

Using the E/C method, size-monodisperse nanocrystals of the salt, $\mathrm{MX}$, should be obtainable from size-monodisperse nanoparticles of the metal, $M$. However, metal nanoparticles that are synthesized electrochemically are not size monodisperse, ${ }^{17,19,26}$ even though these particles nucleate instantaneously, and grow at the diffusion controlled rate. The question is, why?

Brownian dynamics (BD) computer simulations of metal particle growth have helped us understand the origins of particle size dispersion on these surfaces. Briefly, $\mathrm{BD}$ simulations have taught us the following. ${ }^{34}$ At moderate nucleation densities $\left(\sim 10^{10}\right.$ particles $\left.\mathrm{cm}^{-2}\right)$, the growth of neighboring metal particles on a surface (separated by a few hundred angstroms) becomes diffusionally coupled within a few microseconds. At longer times, ${ }^{35}$ the diffusion-controlled flux of reactant (i.e., $\mathrm{M}^{\mathrm{n}+}$ ) for every particle depends on the number and proximity of neighboring particles. Since nucleation is spatially random, the number and proximity of neighbors is different for every particle on the surface, and the diffusion-controlled flux of $\mathrm{M}^{\mathrm{n}+}$ at each growing metal particle is similarly inhomogeneous. This flux inhomogeneity provides the dominant mechanism by which particle size dispersion develops when the nucleation of particles is instantaneous. ${ }^{34}$

In principle, this mechanism of distribution broadening can be defeated by periodically interrupting the growth of metal particles. ${ }^{34}$ During these interruptions, the solution near the electrode surface is permitted to diffusionally mix for a second or so. During this interval, the depletion layer adjacent to each particle is erased, thereby "decoupling" the growth of each nanoparticle on the surface from its nearest neighbors. ${ }^{34,36}$ Decoupling should increase the homogeneity of the reactant flux from particle to particle on the surface, thereby improving the metal particle size monodispersity and the particle size monodispersity for the semiconductor quantum dots derived from these metal nanoparticles. ${ }^{36}$

Since the PL emission lines for ensembles of quantum dots are inhomogeneously broadened as a consequence of particle size inhomogeneity, the PL emission line for 

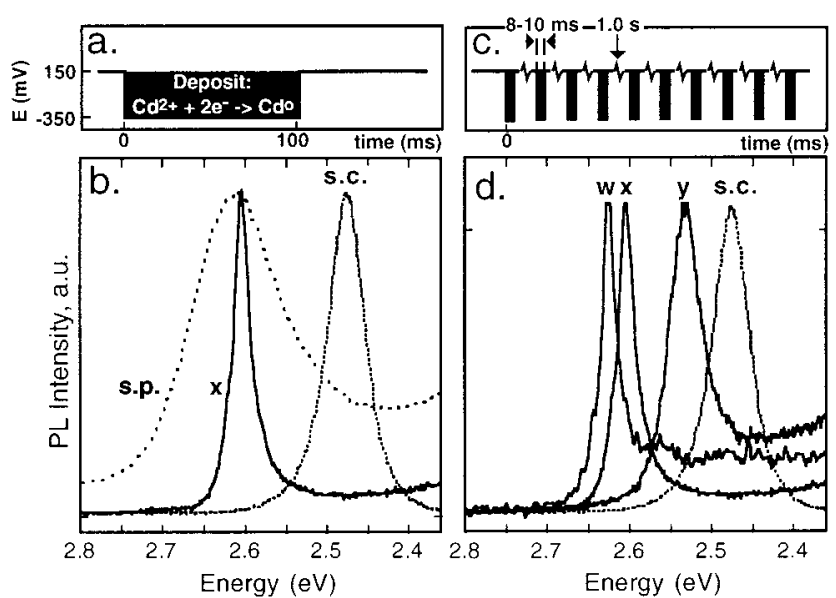

FIGURE 8. Photoluminescence spectra of $\mathrm{CdS} / \mathrm{S}$ nanocrystals on the graphite (0001) surface at $20 \mathrm{~K}$ using $h v_{\mathrm{ex}}=3.53 \mathrm{eV}$. $(\mathrm{a}, \mathrm{c})$ Two voltage pulse programs employed for the electrodeposition of cadmium nanoparticles. (b) Comparison of $\mathrm{CdS} / \mathrm{S}$ nanocrystals prepared from $\mathrm{Cd}^{\circ}$ nanocrystals deposited using a single, 100-ms plating pulse (spectrum "s.p."), multipulse CdS/S nanocrystals deposited using a sequence of $10 \times 10 \mathrm{~ms} \mathrm{Cd}^{\circ}$ plating pulses (spectrum " $x$ "), and a macroscopic (0001) oriented CdS single crystal (spectrum "s.c."). (d) Comparison of three $\mathrm{CdS} / \mathrm{S}$ samples prepared using the multipulse method with $\mathrm{Cd}^{\circ}$ plating programs of $10 \times 8$ ms (spectrum " $w$ "), $10 \times 10 \mathrm{~ms}$ (spectrum " $\mathrm{x}$ "), and, $10 \times 12 \mathrm{~ms}$ (spectrum " $y$ ").

ensembles of quantum dots will become narrower as the particle size distribution becomes narrower. Sasha Gorer investigated the effect of decoupling on the PL emission properties of cadmium sulfide nanocrystals prepared using the E/C method. Typically, the PL emission lines which are observed for thousands of E/C-synthesized $\mathrm{Cul}^{20}$ and $\mathrm{CdS}^{23}$ nanocrystals are 50-150 meV wide (i.e., the full width taken at half the maximum intensity). Shown in Figure 6, for example, is a PL emission spectrum for approximately 300 000, 80- $\AA$-diameter CdS nanocrystals having a width of $125 \mathrm{meV} .{ }^{36} \mathrm{~A}$ single CdS nanocrystal, however, exhibits a much narrower PL emission line width of $\sim 15 \mathrm{meV} .{ }^{37}$ This provides an estimate of the homogeneous line width that should be observed in PL spectra of thousands of identical CdS nanoparticles. The effect of decoupling on the PL spectrum for CdS is shown in Figure 8. In this experiment, Gorer applied ten, 10-ms cadmium plating pulses and spaced (or decoupled) each growth pulse by $1 \mathrm{~s}$ (during which the electrode was at open circuit). These cadmium precursor particles were then converted into $\mathrm{CdS}$, and the PL emission from these nanoparticles was measured. The 18-meV line is dramatically narrower than the $125-\mathrm{meV}$ line for CdS nanoparticles of the same mean diameter obtained from cadmium precursor particles deposited using a single $\mathrm{Cd}$ plating pulse. The size of CdS quantum dots prepared using this "multipulse" synthesis method, and therefore the emission energy, can be adjusted by tuning the pulse duration, as shown in Figure $8{ }^{36}$ This result demonstrates the efficacy of decoupling as a strategy for improving the size monodispersity of semiconductor nanocrystals obtained using the E/C method.

\section{Scheme 3. Schematic Diagram of a Photodetector Based on E/C-Synthesized CdS Nanocrystals on Graphite}
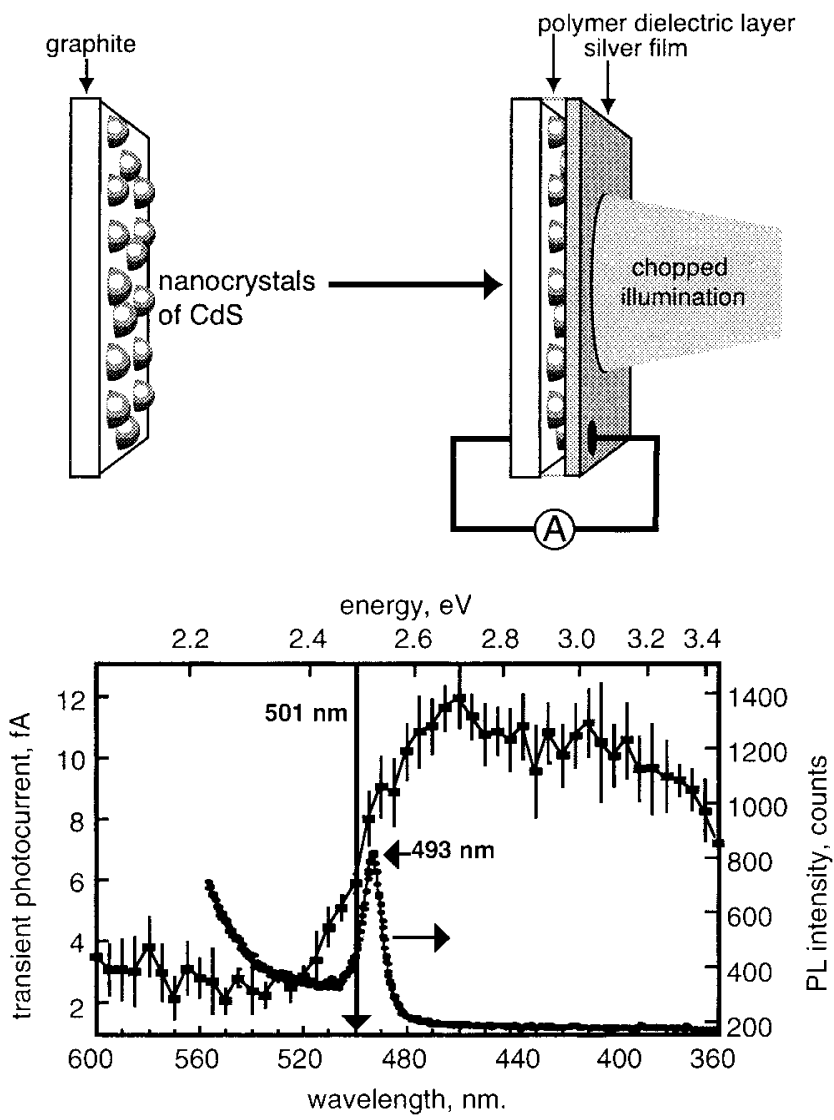

FIGURE 9. Experimental data for the photodetector shown in Scheme 3. Both photoluminescence spectra (acquired at $20 \mathrm{~K}$ using $h v_{\mathrm{ex}}=3.51 \mathrm{eV} ; \mathbf{0}$ ) and device response spectra (acquired at $298 \mathrm{~K}$; च) are shown. The device response was measured by chopping the incident illumination and synchronously measuring the photocurrent. Error bars represent the dispersion of these photocurrent measurements.

\section{A Photodetector, and the Future}

As indicated in the Introduction, quantum dots that are disposed in direct electrical contact with a conductive surface are ideal for device applications. Georg Erley recently demonstrated ${ }^{37}$ the feasibility of electrically detecting the absorption of light by a submonolayer of cadmium sulfide quantum dots supported on a graphite surface. The design of his photodetector is shown in Scheme 3.

Low-temperature PL spectra and transient photocurrent device response spectra for this device are compared in the plot of Figure 9 for a device constructed using $r=$ $80 \AA$ CdS quantum dots. ${ }^{37}$ The PL spectrum for this device exhibits a slight blue shift of the emission maximum to $493 \mathrm{~nm}$, qualitatively as expected for $80-\AA$ CdS quantum dots. The absorption edge seen in the device spectrum was likewise blue-shifted to $501 \mathrm{~nm}$, proving that the photocurrent measurement is sensitive to the nanoparticle band gap even at room temperature. Relative to other types of photodetectors, the quantum dot-based device of Figure 9 is inefficient. However, it serves as proof that E/C-synthesized quantum dots can be exploited to pro- 
duce a device having properties dictated by the electronic properties of the quantum dots.

In principle, quantum dots synthesized using molecular beam epitaxy would also be suitable for the construction of a photodetector like that shown in Scheme 3. In the foreseeable future, we hope to show that that the inverse is also true-i.e., that E/C-synthesized quantum dots can function as substitutes for quantum dots synthesized by molecular beam epitaxy in some applications. We believe the attributes of the E/C method provide a clear motivation for making this substitution. All of the manipulations involved in an E/C synthesis can be performed on a benchtop in a normal laboratory air ambient using inexpensive instrumentation (costing just $\sim \$ 20000$ ) and with amazing speed. The "total synthesis" of Cul, CdS, or $\mathrm{ZnO}$ quantum dots can be carried out in approximately $30 \mathrm{~min}$. The $\mathrm{E} / \mathrm{C}$ method is size selective-a range of different particle sizes can be prepared. And most importantly, the materials and optical properties of the nanocrystals prepared by E/C are on a par with quantum dots prepared using molecular beam epitaxy.

During my nine years at $\mathrm{UCl}$, I have been privileged to work with many talented and enthusiastic co-workers, some of which are mentioned in this article. Presently, semi conductor synthesis investigations in the author's laboratory are funded by the National Science Foundation (DMR-9876479) and the Petroleum Research Fund of theAmerican Chemical Society (33751-AC5). The author also gratefully acknowledges the financial support of the A.P. Sloan Foundation Fellowship, the Camilleand Henry Dreyfus Foundation, theArnold and Mabel Beckman Foundation, and the Procter \& Gamble Corp. Finally, the work described here would have been much more expensive without the generosity of Dr. Art Moore of Advanced Ceramics who has provided my group with much of the graphite used in these experiments.

\section{References}

(1) Rossetti, R.; Nakahara, S.; Brus, L. E. Quantum Size Effects in the Redox Potential, Raman Spectra, and Electron Spectra of CdS Crystallites in Aqueous Solution. J. Chem. Phys. 1984, 79, 10861088.

(2) Rossetti, R.; Ellison, J . L.; Gibson, J . M.; Brus, L. E. Size Effects in Excited Electronic States of Small, Colloidal CdS Crystallites. J . Chem. Phys. 1983, 80, 4464-4469.

(3) Weller, H.; Koch, U.; Gutierrez, M.; Henglein, A. Ber. Bunsen-Ges. Phys. Chem. 1984, 88, 649

(4) Fojtik, A.; Weller, H.; Koch, U.; Henglein, A. PhotoChemistry of Colloidal Metal Sulfides. 8. Photophysics of Extremely Small CdS Particles: Q-State CdS and Magic Agglomeration Numbers. Ber. Bunsen-Ges. Phys. Chem. 1984, 88, 969-977.

(5) Ramsden, J. . .; Gratzel, M. Photoluminescence of Small CdS Particles. J. Chem. Soc., Faraday Trans. 1 1984, 80, 919-933.

(6) Chen, W.; McLendon, G.; Marchetti, A.; Rehm, J . M.; Feedhoff, M. I.; Meyers, C. Size Dependence of Radiative Rate in the Indirect Band Gap Material AgBr. J . Am. Chem. Soc. 1994, 116, 15851586.

(7) Marchetti, A. P.; J ohansson, K. P.; McLendon, G. L. AgBr Photophysics From Optical Studies of Quantum-Confined Crystals. Phys. Rev. B 1993, 47, 4268-4275.

(8) Murray, C. B.; Norris, D. J ; Bawendi, M. G. Synthesis and Characterization of Nearly Monodisperse $\mathrm{CdX}(\mathrm{X}=\mathrm{S}, \mathrm{Se}, \mathrm{Te})$ Semiconductor Nanocrystallites. J. Am. Chem. Soc. 1993, 115, $8706-8715$.

(9) Uchida, H.; Curtis, C. J .; Nozik, A. J . GaAs Nanocrystals Prepared in Quinoline. J. Phys. Chem. 1991, 95, 5382-5384.

(10) Olshavsky, M. A.; Goldstein, A. N.; Alivisatos, A. P. Organometallic Synthesis Of GaAs Crystallites Exhibiting Quantum Confinement. J. Am. Chem. Soc. 1990, 112, 9438-9439.

(11) Wilson, W. L.; Szajowski, P. F.; Brus, L. E. Quantum Confinement In Size-Selected, Surface-Oxidized Silicon Nanocrystals. Science 1993, 262, 1242-1244.
(12) Eaglesham, D. J .; Cerullo, M. Dislocation-Free Stranski-Krastanov Growth of Ge on Si(100). Phys. Rev. Lett. 1990, 64, 1943-1945.

(13) Mo, Y.-W.; Savage, D. E.; Swartzentruber, B. S.; Lagally, M. G. Kinetic Pathway in Stranski-Krastanov Growth of Ge on Si(001). Phys. Rev. Lett. 1990, 65, 1020.

(14) Guha, S.; Madhukar, A.; Raykumar, K. C. Onset of Incoherency and Defect Introduction in the Initial Stages of Molecular Beam Epitaxial Growth of Highly Strained $\operatorname{In}_{x} \mathrm{Ga}_{1-x} \mathrm{As}$ on $\mathrm{GaAs}(100)$. Appl. Phys. Lett. 1990, 57, 2110-2112.

(15) In fact, the growth of semiconductor (e.g., germanium) islands occurs atop a wetting layer of germanium that is several atomic layers in thickness, typically. Undesirable photoluminescence from this wetting layer usually "contaminates" the PL spectrum for MBE-synthesized quantum dots.

(16) As compared with LDs constructed using micrometer-scale emissive layers in which excitons are not confined, an emissive layer based on quantum dots has several advantages: The threshold current $\int^{\text {th }}$ required to induce lasing is reduced, $\int^{\text {th }}$ exhibits less variability with temperature, and the gain bandwidth is narrowed. The application of quantum dots in laser diodes is described in the following publications: Asada, M.; Miyamoto, Y.; Suematsu, Y. IEEE J . Quantum Electron. 1986, QE-22, 1915-1921. Arakawa, Y.; Sakaki, H. Appl. Phys. Lett. 1982, 40, 939-941. Arakawa, Y.; Yariv, A. IEEE J. Quantum Electron. 1985, OE-21, 1666-1674.

(17) Zoval, J . V.; Stiger, R. M.; Biernacki, P. R.; Penner, R. M. Electrochemical Deposition of Silver Nanocrystallites on the Atomically Smooth Graphite Basal Plane. J. Phys. Chem. 1996, 100, 837-844.

(18) Zangwill, A. Physics at Surfaces; Cambridge University Press: Cambridge, UK, 1988

(19) Zoval, J. V.; Lee, J .; Gorer, S.; Penner, R. M. Electrochemical Preparation of Platinum Nanocrystallites With Size Selectivity on Basal Plane-Oriented Graphite Surfaces. J . Phys. Chem. B 1998, 102, 1166-1175.

(20) Hsiao, G. S.; Anderson, M. G.; Gorer, S.; Harris, D.; Penner, R. M. Hybrid Electrochemical/Chemical Synthesis of Supported, Luminescent Semiconductor Nanocrystallites with Size Selectivity: Copper(I)iodide. J. Am. Chem. Soc. 1997, 119, 1439-1448.

(21) Zach, M.; Penner, R. M. Electrodeposition of Size-Monodisperse Nickel Nano- and Micro-Particles on Graphite Using Hydrogen Co-Evolution. Adv. Mater. 1999, submitted for publication.

(22) Anderson, M. A.; Gorer, S.; Penner, R. M. A Hybrid Electrochemi$\mathrm{cal} /$ Chemical Synthesis of Supported, Luminescent Cadmium Sulfide Nanocrystals. J. Phys. Chem. B 1997, 101, 5895-5899.

(23) Gorer, S.; Ganske, J . A.; Hemminger, J . C.; Penner, R. M. The SizeSelective Electrochemical/Chemical Synthesis of Sulfur Passivated Cadmium Sulfide Nanocrystals on Graphite. J. Am. Chem. Soc. 1998, 120, 9584-9593.

(24) Nyffenegger, R. M.; Craft, B.; Shaaban, M.; Gorer, S.; Penner, R. M. A Hybrid Electrochemical/Chemical Synthesis of Zinc Oxide Nanoparticles And Optically Intrinsic Thin Films. Chem. Mater. 1998, 10, 1120-1129.

(25) Morcos, I. Surface-Tension of Stress-Annealed Pyrolytic Graphite. J. Chem. Phys. 1972, 57, 1801-1805.

(26) Stiger, R. M.; Gorer, S.; Craft, B.; Penner, R. M. Investigations of Electrochemical Silver Nanocrystal Growth on Hydrogen-Terminated Silicon(100). Langmuir 1999, 15, 790-798.

(27) Metal surfaces are known to efficiently quench photoluminescence from adsorbed molecules via an energy-transfer mechanism (two relevant reviews are the following: Chance, R. R.; Prock, A.; Silbey, R. Molecular Fluorescence and Energy Transfer at Interfaces. Adv. Chem. Phys. 1978, 37, 1-65. Ford, G. W. Weber, W. H. Electromagnetic Interactions of Molecules with Metal Surfaces. Phys. Rep. 1984, 113, 195-287). A similar effect has been observed for chromophores at semiconductor surfaces where quenching is dominated instead by electron transfer (two relevant publications are the following: Hashimoto, K.; Hiramoto, M.; Sakata, T. Photoinduced Electron Transfer From Adsorbed Rhodamine B To Oxide Semiconductor Substrates in Vacuo: Semiconductor Dependence. Chem. Phys. Lett. 1988, 148, 215220. Suto, S.; Uchida, W.; Yashima, M.; Goto, T. Luminescence Quenching of an Ultrathin Tetraphenylporphyrin Film on a Conductive $\mathrm{SnO}_{2}$ Substrate. Phys. Rev. B 1987, 35, 4393-4397)

(28) The quantum yield for E/C-synthesized nanocrystals has not been directly measured because it has not been possible, so far, to obtain absorption spectra for these nanoparticles on the absorbing graphite surface. It is probable that the PL quantum yield for quantum dots on the graphite surface is reduced by both of the mechanisms discussed in ref 27

(29) So far we have obtained photoluminescence spectra for $\mathrm{ZnO}$ films, but not for nanoparticles because photons having the higher energy necessary to excite fluorescence in quantumconfined $\mathrm{ZnO}$ nanoparticles have not been available in our laboratory. 
(30) The graphite surface may also play a role in reducing the intensity of trap-state emission for $\mathrm{ZnO}$. The radiative lifetime for trap states in $\mathrm{ZnO}$ is much greater than the lifetime for band-edge emission, and emission from these midgap states would therefore be preferentially quenched relative to band-edge emission.

(31) Bawendi, M. G.; Steigerwald, M. L.; Brus, L. E. The Quantum Mechanics of Larger Semiconductor Clusters (Quantum Dots). Annu. Rev. Phys. Chem. 1990, 41, 477-496.

(32) Steigerwald, M. L.; Brus, L. E. Synthesis, Stabilization, and Electronic Structure Of Quantum Semiconductor Nanoclusters. Annu. Rev. Mater. Sci. 1989, 19, 471-495.

(33) Steigerwald, M. L.; Brus, L. E. Semiconductor Crystallites-a Class Of Large Molecules. Acc. Chem. Res. 1990, 23, 183-188.

(34) Fransaer, J .; Penner, R. M. Brown Dynamics Simulations of the Growth of metal Nanocrystal Ensembles on Electrodes Surfaces
From Solution. I. Instantaneous Nucleation and DiffusionControlled Growth. J. Phys. Chem. B 1999, 103, 7643.

(35) The diffusion-controlled growth of 20-Å-diameter metal nanoparticles at a density of $10^{10} \mathrm{~cm}^{-2}$ from a $1.0 \mathrm{mM}$ solution of metal ions typically requires $10-20 \mathrm{~ms}$.

(36) Gorer, S.; Penner, R. M. "Multipulse" Electrochemical/Chemical Synthesis of CdS/S Core-Shell Nanocrystals Exhibiting Ultranarrow Photoluminescence Emission Lines. J . Phys. Chem. B 1999, 103, 5750-5753.

(37) Erley, G.; Gorer, S.; Penner, R. M. Transient Photocurrent Spectroscopy: Electrical Detection of Optical Absorption For Supported Semiconductor Nanocrystals in a Simple Device Geometry. Appl. Phys. Lett. 1998, 72, 2301-2303.

AR9702839 